\title{
Perturbative subtraction of lattice artifacts in the computation of renormalization constants
}

\author{
M. Constantinou ${ }^{a}$, M. Costa ${ }^{a}$, M. Göckeler $^{b}$, R. Horsley ${ }^{c}$, H. Panagopoulos ${ }^{a}$, \\ H. Perlt ${ }^{* d}$, P. E. L. Rakow ${ }^{e}$, G. Schierholz ${ }^{f}$, A. Schiller ${ }^{d}$ \\ ${ }^{a}$ Department of Physics, University of Cyprus, Nicosia, CY-1678, Cyprus \\ ${ }^{b}$ Institut für Theoretische Physik, Universität Regensburg, 93040 Regensburg, Germany \\ ${ }^{c}$ School of Physics, University of Edinburgh, Edinburgh EH9 3JZ, UK \\ ${ }^{d}$ Institut für Theoretische Physik, Universität Leipzig, 04109 Leipzig, Germany \\ ${ }^{e}$ Theoretical Physics Division, Department of Mathematical Sciences, University of Liverpool, \\ Liverpool L69 3BX, UK \\ ${ }^{f}$ Deutsches Elektronen-Synchrotron DESY, 22603 Hamburg, Germany \\ E-mail: perlt@itp.uni-leipzig.de
}

\begin{abstract}
The determination of renormalization factors is of crucial importance. They relate the observables obtained on finite, discrete lattices to their measured counterparts in the continuum in a suitable renormalization scheme. Therefore, they have to be computed as precisely as possible. A widely used approach is the nonperturbative Rome-Southampton method. It requires, however, a careful treatment of lattice artifacts. They are always present because simulations are done at lattice spacings $a$ and momenta $p$ with $a p$ not necessarily small. In this paper we try to suppress these artifacts by subtraction of one-loop contributions in lattice perturbation theory. We compare results obtained from a complete one-loop subtraction with those calculated for a subtraction of $O\left(a^{2}\right)$.
\end{abstract}

The 30th International Symposium on Lattice Field Theory

June 24 - 29, 2012

Cairns, Australia

\footnotetext{
* Speaker.
} 


\section{Introduction}

Renormalization factors relate observables computed on finite lattices to their continuum counterparts in specific renormalization schemes. Therefore, their determination should be as precise as possible in order to allow for a reliable comparison with experimental results. One approach is based on lattice perturbation theory [四]. However, it suffers from its intrinsic complexity, slow convergence and the impossibility to handle mixing with lower-dimensional operators. Therefore, nonperturbative methods have been developed in the last years. Among them the so-called Rome-Southampton method [ [వ] (or RI-MOM scheme) is widely used because of its simple implementation. It requires, however, gauge fixing.

In a recent paper [B] some of us have given a comprehensive discussion and comparison of perturbative and nonperturbative renormalization. One of the conclusions was the possibility to suppress the unavoidable lattice artifacts by subtracting them perturbatively. For simple operators this can be done in one-loop order completely by computing the corresponding diagrams numerically. While being very effective this procedure is rather involved and not suited as a general method for more complex operators, especially for operators with more than one covariant derivative. A more general approach could be based on the subtraction of one-loop terms of the order $a^{2}$ with $a$ being the lattice spacing. The computation of those terms has been pioneered by the Cyprus group [ [ 4 ] and applied to various operators for different actions.

In this paper we apply this "reduced" subtraction procedure to some exemplary operators and compare the results with those of the complete one-loop subtraction as given in [3]]. We investigate the point operators $\mathscr{O}^{\mathrm{S}}=\bar{u} d, \mathscr{O}_{\mu}^{\mathrm{V}}=\bar{u} \gamma_{\mu} d, \mathscr{O}_{\mu}^{\mathrm{A}}=\bar{u} \gamma_{5} \gamma_{\mu} d$ and $\mathscr{O}_{\mu \nu}^{\mathrm{T}}=\bar{u} \sigma_{\mu \nu} d$ for light quarks $(u, d)$. The corresponding $\mathrm{Z}$ factors have been measured (and chirally extrapolated) at $\beta=5.20,5.25,5.29$ and 5.40. We used clover improved Wilson fermions with plaquette gauge action. All results are computed in Landau gauge. The clover parameter $c_{S W}$ used in the perturbative calculation is set to its lowest order value $c_{S W}=1$.

\section{Renormalization group invariant operators}

In the RI-MOM scheme the renormalization constant $Z$ is obtained by imposing the condition

$$
\frac{1}{12} \operatorname{tr}\left(\Gamma_{R}(p) \Gamma_{\text {Born }}^{-1}(p)\right)=1
$$

at $p^{2}=\mu^{2}$, where $\Gamma$ is the corresponding amputated Green function of the operator $\mathscr{O}$ under study. The $\mathrm{Z}$ factors relate the renormalized to the unrenormalized Green functions

$$
\Gamma_{R}(p)=Z_{q}^{-1} Z \Gamma(p),
$$

with $Z_{q}$ being the quark field renormalization constant determined by

$$
Z_{q}(p)=\frac{\operatorname{tr}\left(-i \sum_{\lambda} \gamma_{\lambda} \sin \left(a p_{\lambda}\right) a S^{-1}(p)\right)}{12 \sum_{\lambda} \sin ^{2}\left(a p_{\lambda}\right)},
$$

( $S^{-1}$ is the inverse quark propagator). Using (ㅁ. $(\mathbb{C})$ we compute $Z$ from

$$
Z_{q}^{-1} Z \frac{1}{12} \operatorname{tr}\left(\Gamma(p) \Gamma_{\text {Born }}^{-1}(p)\right)=1 .
$$


For operators which carry at least one space-time index (i.e. the corresponding $O(4)-$ multiplet has dimension greater than 1) we use an averaging procedure as described in [3] .

We define the so-called renormalization group invariant (RGI) operator, which is independent of scale $M$ and scheme $\mathscr{S}$, by [B]]

$$
\mathscr{O}^{\mathrm{RGI}}=\Delta Z^{\mathscr{S}}(M) \mathscr{O}^{\mathscr{S}}(M)=Z^{\mathrm{RGI}}(a) \mathscr{O}_{\text {bare }}
$$

with

$$
\Delta Z^{\mathscr{S}}(M)=\left(2 \beta_{0} \frac{g^{\mathscr{S}}(M)^{2}}{16 \pi^{2}}\right)^{-\left(\gamma_{0} / 2 \beta_{0}\right)} \exp \left\{\int_{0}^{g^{\mathscr{S}}(M)} d g^{\prime}\left(\frac{\gamma^{\mathscr{S}}\left(g^{\prime}\right)}{\beta^{\mathscr{S}}\left(g^{\prime}\right)}+\frac{\gamma_{0}}{\beta_{0} g^{\prime}}\right)\right\}
$$

and

$$
Z^{\mathrm{RGI}}(a)=\Delta Z^{\mathscr{S}}(M) Z_{\mathrm{bare}}^{\mathscr{S}}(M, a) .
$$

$g^{\mathscr{S}}, \gamma^{\mathscr{S}}$ and $\beta^{\mathscr{S}}$ are the coupling constant, the anomalous dimensions and the $\beta$-function in scheme $\mathscr{S}$, respectively ( $\gamma_{0}$ and $\beta_{0}$ are scheme independent and denote the corresponding lowest order coefficients). Relations ([2.5), (L.G) and (L.J) allow us to compute the $\mathrm{Z}$ factor of the operator $\mathscr{O}$

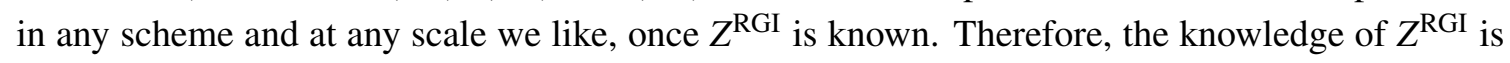
very useful for the renormalization procedure in general. Ideally, $Z^{\mathrm{RGI}}$ depends only on the lattice spacing $a$. Computed on a finite lattice, however, it suffers from lattice artifacts. For a precise determination it is essential to have these discretization errors under control.

Most quantities on the lattice are computed within the so-called RI'-MOM scheme. However, being not covariant, this scheme is not very suitable for computing the anomalous dimensions. Therefore, we replace ([2.]) by

$$
Z^{\mathrm{RGI}}(a)=\Delta Z^{\mathscr{S}}\left(M=\mu_{p}\right) Z_{\mathrm{RI}^{\prime}-\mathrm{MOM}}^{\mathscr{S}}\left(M=\mu_{p}\right) Z_{\mathrm{bare}}^{\mathrm{RI}^{\prime}-\mathrm{MOM}}\left(\mu_{p}, a\right)
$$

For the intermediate scheme $\mathscr{S}$ we have chosen a momentum subtraction scheme. On a lattice with linear extent $L$, the scale $\mu_{P}$ should fulfill the relation

$$
1 / L^{2} \ll \Lambda_{\mathrm{QCD}}^{2} \ll \mu_{p}^{2} \ll 1 / a^{2},
$$

then $Z^{\mathrm{RGI}}(a)$ would be independent of $\mu_{p}$ and from the resulting plateau we could read off the corresponding final value. The formula which is used to compute the perturbative conversion factor $Z_{\mathrm{RI}^{\prime}-\mathrm{MOM}}^{\mathscr{S}}(p)$ is given in [B]] together with all needed coefficients of the $\beta$-function and anomalous dimensions. We will not give them here - the reader is referred to this reference.

\section{Perturbative subtraction of order $a^{2}$}

As shown in [3] the complete one-loop subtraction of lattice artifacts results in a very weak $p$-dependence of the $Z^{\mathrm{RGI}}$ which allows a rather precise determination. In the absence of this procedure there is the question whether a "reduced" subtraction can do a similar job. It could be based on a one-loop calculation including all possible $O\left(a^{2}\right)$ terms performed by the Cyprus group [四]. Recently, it has been demonstrated that for some selected operators and actions the subtraction of those terms shows encouraging results [[]]. 
Let us denote the $O\left(a^{2}\right)$ part of the one-loop contribution to the renormalization constant by $Z_{1-\text { loop }}^{\left(a^{2}\right)}(p, a)$. Then we define the subtracted $\mathrm{Z}$ factor as

$$
Z_{\mathrm{bare}}^{\mathrm{RI}^{\prime}-\mathrm{MOM}}(p, a)_{\mathrm{MC} \text {,sub }}=Z_{\mathrm{bare}}^{\mathrm{RI}^{\prime}-\mathrm{MOM}}(p, a)_{\mathrm{MC}}-g_{\star}^{2} Z_{1-\text { loop }}^{\left(a^{2}\right)}(p, a)
$$

where $g_{\star}$ can be chosen to be either the bare lattice coupling $g$ or the boosted coupling $g_{\mathrm{B}}$ defined by $g_{\mathrm{B}}^{2}=g^{2} / P(g), P(g)$ is the measured plaquette at $g$. The final renormalization group independent $\mathrm{Z}$ factor is then computed from (B.D) using (R.Z), where we expect slightly different numbers depending on the choice of coupling $g_{\star}$. As suggested by the results in [3] we choose $g_{\star}=g_{\mathrm{B}}$. The subtraction terms $Z_{1-\text { loop }}^{\left(a^{2}\right)}(p, a)$ can be calculated to a very high precision. Therefore, the only significant errors to $Z_{\mathrm{bare}}^{\mathrm{RI}^{\prime}-\mathrm{MOM}}(p, a)_{\mathrm{MC} \text {,sub }}$ are due to the Monte Carlo simulations. In Figure $\mathbb{\square}$
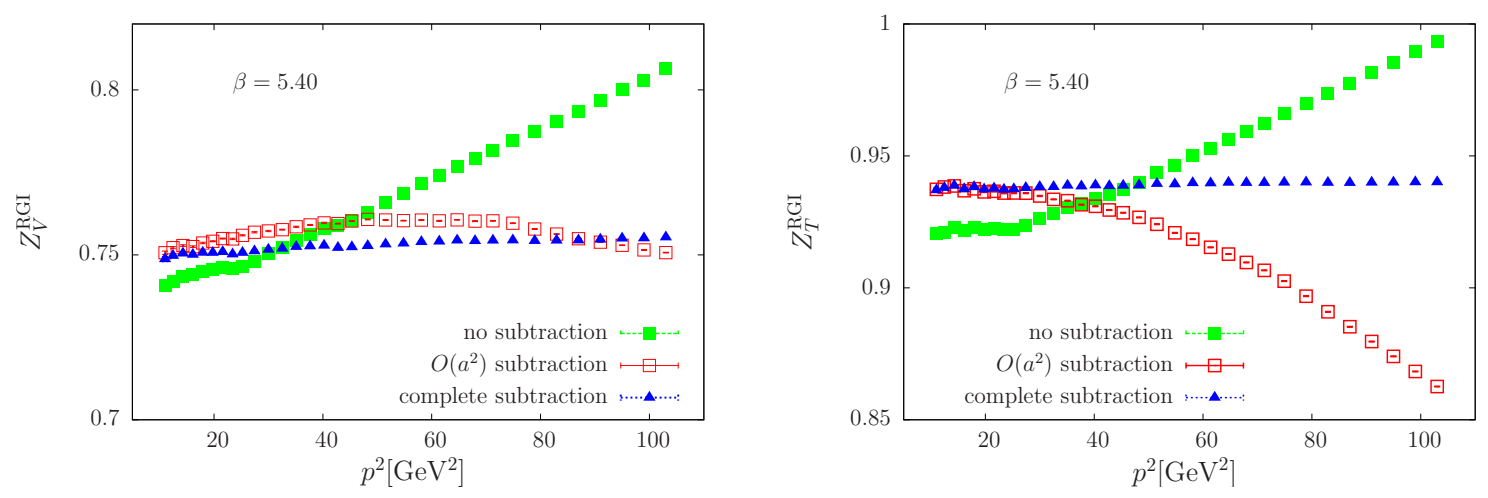

Figure 1: Unsubtracted and subtracted renormalization constants for the vector operator $\mathscr{O}^{V}$ (left) and the tensor operator $\mathscr{O}^{T}$ (right).

we show the effect of subtraction (complete and $O\left(a^{2}\right)$ ) for the vector and tensor operators. The complete one-loop subtraction results in a clear plateau for both $Z^{\mathrm{RGI}}$ factors. Using the $O\left(a^{2}\right)$ subtraction there remains a more or less pronounced curvature. For small $p^{2}$ both subtraction methods agree, as they should.

\section{Fit procedure}

Compared to the complete one-loop subtraction we expect $Z_{\text {bare }}^{\mathrm{RI}^{\prime}-\mathrm{MOM}}(p, a)_{\mathrm{MC} \text {,sub as computed }}$ from (B. 1 ) to contain higher $p^{2 n}(n \geq 2)$ terms constrained only by hypercubic symmetry. Therefore, we parametrize the subtracted data for each $\beta$ in terms of hypercubic structures as follows (see $(\mathbb{R} .8))$

$$
\begin{aligned}
Z_{\mathrm{RI}^{\prime}-\mathrm{MOM}}^{\mathscr{S}}(p) Z_{\mathrm{bare}}^{\mathrm{RI}^{\prime}-\mathrm{MOM}}(p, a)_{\mathrm{MC}, \mathrm{sub}}= & Z^{\mathrm{RGI}}(a) / \Delta Z^{\mathscr{S}}(p)+c_{1} a^{2} S_{2}+c_{2} a^{2} S_{4} / S_{2} \\
& +c_{3} a^{2} S_{6} / S_{2}^{2}+c_{4} a^{4} S_{2}^{2}+c_{5} a^{4} S_{4} \\
& +c_{6} a^{6} S_{2}^{3}+c_{7} a^{6} S_{4} S_{2}+c_{8} a^{6} S_{6},
\end{aligned}
$$

with $S_{n}=\sum_{i}^{4} p_{i}^{n}$. The parameters $c_{1}-c_{8}$ describe the lattice artifacts. Together with the target parameter $Z^{\text {RGI }}(a)$ we have nine parameters for this general case. In view of the limited number of 
data points for each single $\beta$ value $(5.20,5.25,5.29,5.40)$ we apply the ansatz (4.1) to all $\beta$ values simultaneously with

$$
Z^{\mathrm{RGI}}(a) / \Delta Z^{\mathscr{I}}(p) \rightarrow Z_{k}^{\mathrm{RGI}}(a) / \Delta Z_{k}^{\mathscr{S}}(p)
$$

where $k$ labels the corresponding $\beta$ value. The parameters $c_{i}$ are taken to be independent of $\beta$. This enhances the ratio (number of data points)/(number of fit parameters) significantly!

Of course, there is a certain degree of freedom in the fit procedure. One choice regards the interval $p_{\text {min }}^{2} \leq p^{2} \leq p_{\text {max }}^{2}$ used for the fit. Inspection of data and of the results in [3] suggests to use $p_{\min }^{2}=10 \mathrm{GeV}^{2}$ for all $\beta$ values and all considered operators. For $p_{\max }^{2}$ we choose the corresponding maximal available momentum. Another interesting point is to investigate whether the $O\left(a^{2}\right)$ subtraction has been sufficient to subtract (almost) all $p^{2}$ dependence. Therefore, we perform two kinds of fits: one with all hypercubic structures under consideration $\left(Z_{i}^{\mathrm{RGI}}(a), c_{1}, c_{2}, c_{3}, c_{4}, c_{5}\right.$, $\left.c_{6}, c_{7}, c_{8}\right)$ and one with the $a^{2}$ dependence omitted $\left(Z_{i}^{\mathrm{RGI}}(a), c_{4}, c_{5}, c_{6}, c_{7}, c_{8}\right)$.

Additionally, the renormalization factors are influenced by the choice for $r_{0} \Lambda_{\overline{\mathrm{MS}}}$. This quantity enters $\Delta Z^{\mathscr{S}}(M)$ in $\left(\mathbb{R} \cdot(\mathbb{G})\right.$ via the corresponding coupling $g^{\mathscr{S}}(M)$ (for details see [B]]). We use two values: as standard value we take $r_{0} \Lambda_{\overline{\mathrm{MS}}}=0.73$, suggested by [目], as a second value we choose $r_{0} \Lambda_{\overline{\mathrm{MS}}}=0.78$, close to the result given in [0]. The Sommer scale $r_{0}$ is chosen as $r_{0}=0.5 \mathrm{fm}$. The relation between the lattice spacing $a$ and the inverse lattice coupling $\beta$ is given by $r_{0} / a=$ 6.050 $(\beta=5.20), 6.603(\beta=5.25), 6.983(\beta=5.29)$ and $8.285(\beta=5.40)$ [ [ $\beta]$ ].

\section{Results}

In Figure $\square$ we show the results for our fit procedures for the choice $r_{0} \Lambda_{\overline{\mathrm{MS}}}=0.73$. The $Z^{\text {RGI }}$ factors for the operators $V$ and $T$ coincide within errors well with the complete one-loop subtraction calculation. The renormalization factors for $S$ and $A$ differ more significantly. Now, we compare the different fit procedures using the perturbatively subtracted $O\left(a^{2}\right)$ contributions (see (B.])). Generally, one can state that fitting only $O\left(a^{4}, a^{6}\right)$ structures still leads to reasonable results with smaller errors. This would mean that the subtraction of one-loop $O\left(a^{2}\right)$ terms takes into account (almost) all lattice artifacts proportional to $a^{2}$. In addition, we do not find (at least for the operators considered) a remarkable difference using either a single fit for each individual $\beta$ data set or a combined fit for all four $\beta$ values.

In order to estimate the quality of the fit we compute the relative difference

$$
\delta Z(p)=\left(Z_{\text {data }}(p)-Z_{\mathrm{fit}}(p)\right) / Z_{\mathrm{data}}(p),
$$

where $Z_{\mathrm{data}}(p)$ are the data for $Z_{\mathrm{RI}^{\prime}-\mathrm{MOM}}^{\mathscr{S}}(p) Z_{\mathrm{bare}}^{\mathrm{RI}-\mathrm{MOM}}(p, a)_{\mathrm{MC}, \text { sub. }} Z_{\mathrm{fit}}(p)$ is the result of the corresponding fit. In Figure $\mathbb{B}$ we show these differences for the operators $\mathscr{O}^{S}$ and $\mathscr{O}^{T}$ (the other $\mathrm{Z}$ factors for different $\beta$ behave similarly). One recognizes that the $\delta Z(p)$ are essentially in the per mill range. Moreover, the figures suggest that the fit to $O\left(a^{4}, a^{6}\right)$ structures seems to be sufficient compared to fitting all structures in (4.).

In Table $\square$ we give the results for a fit to the higher order lattice artifact terms $O\left(a^{4}, a^{6}\right)$, where the $Z^{\text {RGI }}$ are obtained from each single $\beta$ data set. The results are given in the form value(err1)(err2), where err 1 is the error of the fit parameters in the applied nonlinear fit algorithm. err 2 denotes the change in the results if one uses the fit range $8 \mathrm{GeV}^{2} \leq p^{2}$ which is not totally excluded. It can serve as an indicator of systematic error. 

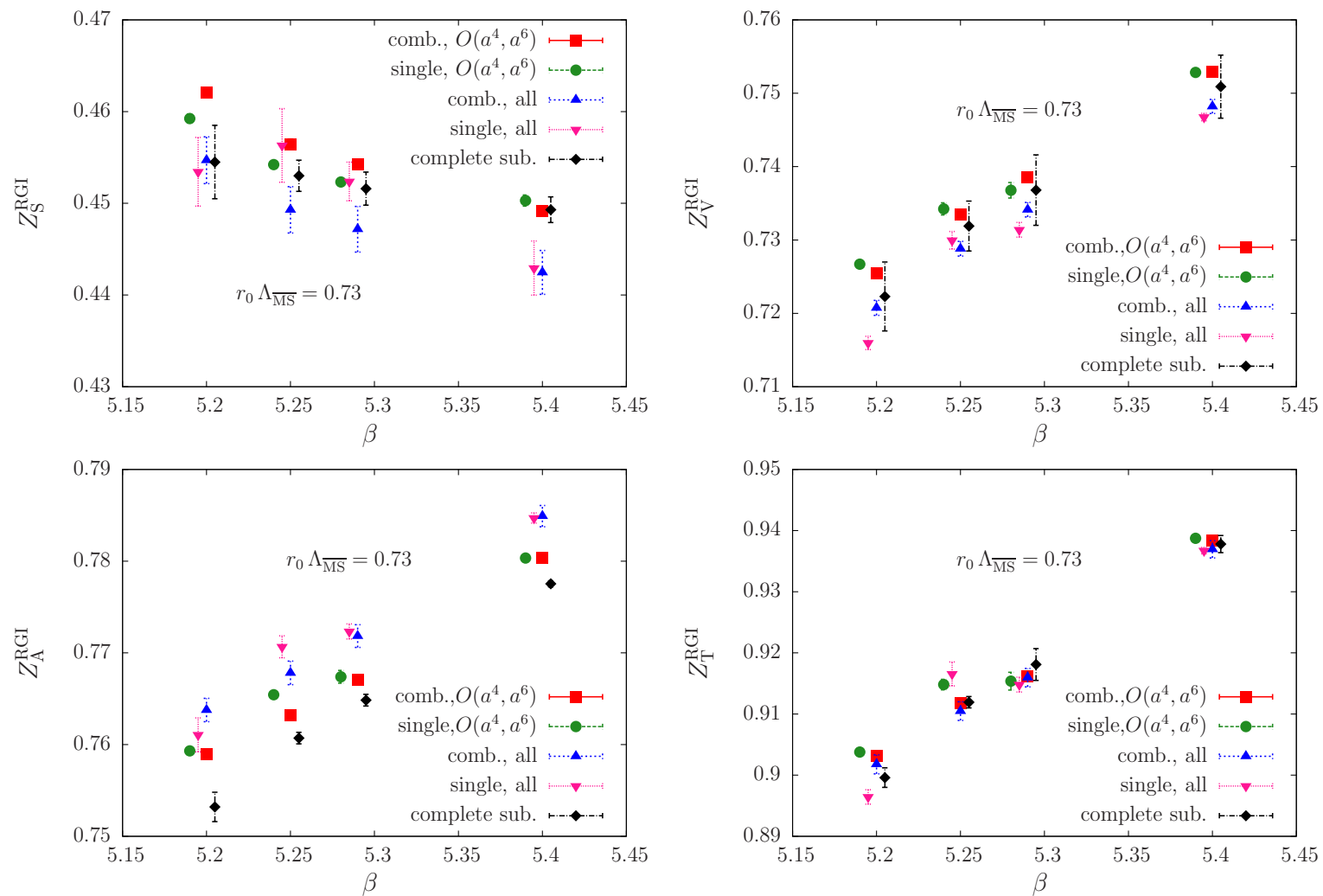

Figure 2: $Z^{\mathrm{RGI}}$ for the local point operators $S, V, A$ and $T$ at $r_{0} \Lambda_{\overline{\mathrm{MS}}}=0.73$. The legends denote: "comb." uses the fit ansatz (4. (ل) + (4.2), "single" is based on (4. (ل) for each $\beta$, "all" fits the general hypercubic structure and " $O\left(a^{4}, a^{6}\right)$ " only possible $a^{4}$ and $a^{6}$ parts. The data points for "complete sub." are obtained from the fit procedure discussed in [B]]; they serve as reference.
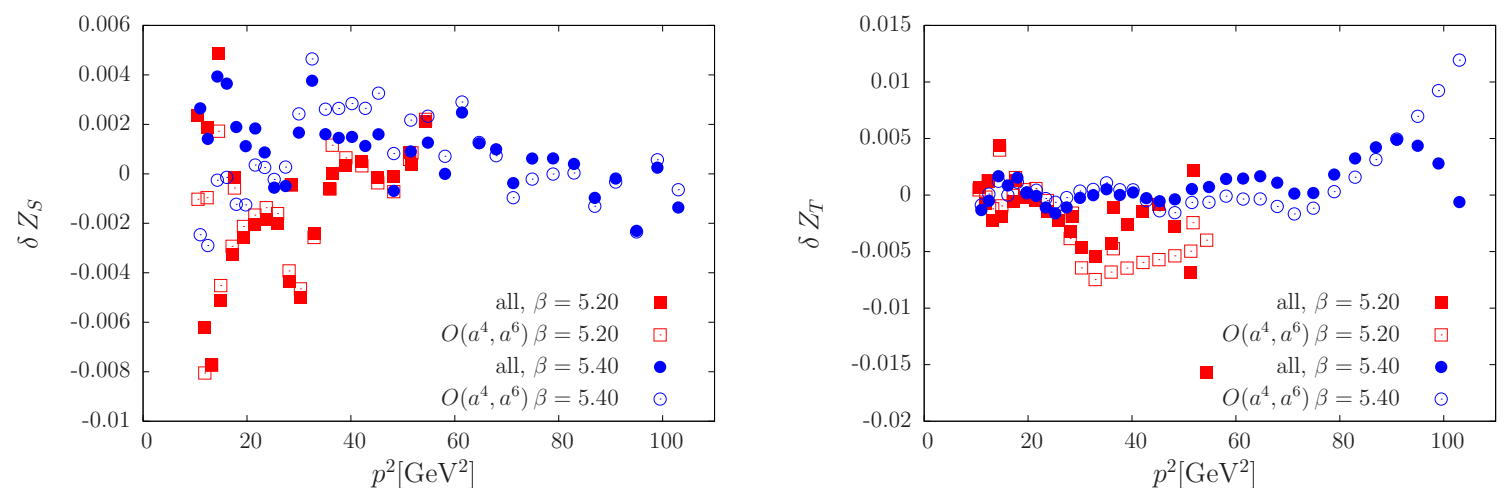

Figure 3: Relative errors $\delta Z$ for the scalar operator $\mathscr{O}^{S}$ (left) and the tensor operator $\mathscr{O}^{T}$ (right) for $r_{0} \Lambda_{\overline{\mathrm{MS}}}=$ 0.73. "all" denotes the fit to the general hypercubic structure and " $O\left(a^{4}, a^{6}\right)$ " only to possible $a^{4}$ and $a^{6}$ parts.

Our fit procedure suggests that a "reduced" perturbative subtraction with a subsequent fit of $O\left(a^{4}, a^{6}\right)$ lattice artifacts leads to reliable results. This algorithm can be used for operators with higher numbers of derivatives where a complete one-loop subtraction is not available. However, it 


\begin{tabular}{|c|c|l|l|l|l|}
\hline Op. & $r_{0} \Lambda_{\overline{\mathrm{MS}}}$ & $\left.Z^{\mathrm{RGI}}\right|_{\beta=5.20}$ & $\left.Z^{\mathrm{RGI}}\right|_{\beta=5.25}$ & $\left.Z^{\mathrm{RGI}}\right|_{\beta=5.29}$ & $\left.Z^{\mathrm{RGI}}\right|_{\beta=5.40}$ \\
\hline $\mathscr{O}^{\mathrm{S}}$ & 0.73 & $0.4592(5)(1)$ & $0.4542(3)(3)$ & $0.4523(3)(3)$ & $0.4503(7)(1)$ \\
$\mathscr{O}^{\mathrm{V}}$ & 0.73 & $0.7267(5)(3)$ & $0.7342(9)(5)$ & $0.7368(11)(7)$ & $0.7528(4)(5)$ \\
$\mathscr{O}^{\mathrm{A}}$ & 0.73 & $0.7593(4)(-11)$ & $0.7654(4)(-1)$ & $0.7674(7)(-1)$ & $0.7803(3)(-4)$ \\
$\mathscr{O}^{\mathrm{T}}$ & 0.73 & $0.9038(3)(1)$ & $0.9148(9)(21)$ & $0.9154(15)(9)$ & $0.9387(3)(2)$ \\
\hline $\mathscr{O}^{\mathrm{S}}$ & 0.78 & $0.4699(6)(-4)$ & $0.4650(6)(-2)$ & $0.4633(5)(-2)$ & $0.4579(6)(-4)$ \\
$\mathscr{O}^{\mathrm{V}}$ & 0.78 & $0.7265(5)(4)$ & $0.7340(9)(5)$ & $0.7366(11)(7)$ & $0.7527(5)(5)$ \\
$\mathscr{O}^{\mathrm{A}}$ & 0.78 & $0.7591(4)(-12)$ & $0.7652(4)(0)$ & $0.7672(7)(-1)$ & $0.7802(3)(-4)$ \\
$\mathscr{O}^{\mathrm{T}}$ & 0.78 & $0.8910(6)(13)$ & $0.9042(11)(26)$ & $0.9085(18)(11)$ & $0.9332(4)(7)$ \\
\hline
\end{tabular}

Table 1: $Z^{\text {RGI }}$ for the point-like operators under consideration. The results are obtained from a fit to $O\left(a^{4}, a^{6}\right)$ structures and for each single $\beta$ data set. The fit range in momentum space is $10 \mathrm{GeV}^{2} \leq p^{2}$. The results are given in the form value (err 1$)($ err 2$)$ where err 1 denotes the error from the nonlinear fit. err 2 is the shift to value if the fit is performed for $8 \mathrm{GeV}^{2} \leq p^{2}$. The shown numbers for value correspond to the (green) full circles in Figure ฉ.

requires a careful investigation of the prerequisites and the parameter choices for each new operator. Finally, one should add that the computation of the $O\left(g^{2} a^{2}\right)$ terms in a one-loop calculation for those operators is also challenging.

This investigation has been supported partly by DFG under contract SCHI 422/8-1, by the EU grant 283286 (HadronPhysics3), SFB/TRR55 (Hadron Physics from Lattice QCD) and by RPF(Cyprus) grant NatSci/0311.

\section{References}

[1] S. Capitani, Phys. Rept. 382 (2003) 113 [arXiv:hep-lat/0211036].

[2] G. Martinelli, C. Pittori, C. T. Sachrajda, M. Testa and A. Vladikas, Nucl. Phys. B 445 (1995) 81 [arXiv:hep-lat/9411010].

[3] M. Göckeler, R. Horsley, Y. Nakamura, H. Perlt, D. Pleiter, P. E. L. Rakow, A. Schäfer, G. Schierholz, A. Schiller, H. Stüben and J. M. Zanotti, Phys. Rev. D 82 (2010) 114511 arXiv:1003.5756 hep-lat].

[4] M. Constantinou, V. Lubicz, H. Panagopoulos and F. Stylianou, JHEP 0910 (2009) 064 [arXiv:0907.0381 hep-lat].

[5] C. Alexandrou, M. Constantinou, T. Korzec, H. Panagopoulos and F. Stylianou, Phys. Rev. D 86 (2012) 014505 arXiv:1201.5025 hep-lat].

[6] B. Leder et al. [ALPHA Collaboration], PoS LATTICE 2010, 233 (2010) arXiv:1012.1141 hep-lat].

[7] P. Fritzsch, F. Knechtli, B. Leder, M. Marinkovic, S. Schaefer, R. Sommer and F. Virotta, Nucl. Phys. B 865 (2012) 397 [arXiv: 1205.5380hep-lat]].

[8] G. Bali and J. Najjar (2012), in preparation. 